\title{
Les erreurs fréquentes des étudiants universitaires hispanophones de FLE à l'écrit
}

\author{
Common writing mistakes of the Spanish-speaking \\ university students of French as a foreign language
}

\author{
MARC VIÉMON \\ Université de Séville \\ mviemon@us.es
}

\begin{abstract}
In this article, we classify the most common morphosyntactic mistakes that Spanish-speaking university students of French as a foreign language at level A2-B1 make when writing. We will focus solely on the area of morphosyntax as it is always where the most mistakes are made. Furthermore, we will discuss in more detail the issue of the agreement, as it is the most common error within our corpus. We conclude that the mispronunciation of the learners as well as bad reading habits could be the origin of a certain number of morphological errors. Finally, we suggest some corrective practices aiming to reduce, or even eradicate, the detected errors: autocorrection, collective correction, and rereading.
\end{abstract}

\section{Keywords}

error analysis, french language, spanish-speaking students, writing, corrective techniques.

\section{Resumen}

En este artículo presentamos la clasificación de los errores morfosintácticos más frecuentes cometidos por los estudiantes universitarios hispanohablantes de francés lengua extranjera en sus producciones escritas. Solo consideramos el área morfosintáctica por ser esta la que reúne siempre más cantidad de errores. Asimismo, abordamos con más detenimiento la cuestión de la ausencia de concordancia, que es el error más común en nuestro corpus. Llegamos a la conclusión de que la pronunciación defectuosa de los alumnos, así como malos hábitos de lectura podrían ser una posible fuente de estos errores morfológicos. Finalmente, proponemos algunas prácticas correctivas con el fin de reducir, e incluso eliminar, los errores detectados: autocorrección, corrección colectiva y relectura.

\section{Palabras clave}

análisis de errores, lengua francesa, estudiantes hispanohablantes, producción escrita, técnicas correctivas. 


\section{Introduction}

Les recherches exposées dans cet article ont pour objectif de contribuer à améliorer, chez les apprenants hispanophones, la maîtrise grammaticale de la langue française à l'écrit. Elles ont été motivées par notre propre expérience comme enseignant de langue française au niveau universitaire durant laquelle nous avons constaté une déficience de la maîtrise grammaticale sur des erreurs bien particulières et récurrentes, non seulement dans les premiers niveaux, mais également chez des étudiants de niveaux plus avancés, ce qui est plus grave étant donné que les étudiants en question, pour beaucoup d'entre eux, sont ensuite censés enseigner eux-mêmes le français dans le primaire et le secondaire.

Actuellement, les étudiants universitaires évoluent dans un système éducatif dans lequel, on le sait, prédominent l'approche communicative (Bérard, 1991) et la perspective actionnelle (Puren, 2006) pour l'apprentissage des langues étrangères, avec, comme modèle à suivre, le Cadre Européen Commun de Référence (CECR) et les différents niveaux de compétence langagière allant de $\mathrm{A} 1$ à $\mathrm{C} 2$. Dans cet ouvrage de référence on défend que l'objectif principal de l'apprentissage d'une langue étrangère -ou de plusieurs, puisque l'approche défendue est aussi plurilingue et pluriculturelle- est de pouvoir communiquer (CECR, 2001: 9) et plus généralement interagir (CECR Volume complémentaire, 2018: 23) dans cette langue.

Ainsi, pour évaluer le niveau de langue des apprenants dans leurs productions écrites, le CECR propose, en ce qui concerne la correction grammaticale des niveaux A2 et B1, les descripteurs suivants:

A2: Peut utiliser des structures simples correctement mais commet encore systématiquement des erreurs élémentaires comme, par exemple, la confusion des temps et l'oubli de l'accord. Cependant le sens général reste clair. (CECR, 2001: 90)

B1: Communique avec une correction suffisante dans des contextes familiers; en règle générale, a un bon contrôle grammatical malgré de nettes influences de la langue maternelle. Des erreurs peuvent se produire mais le sens général reste clair. (CECR, 2001: $90)^{1}$

Comme on le voit, ce qui est important est que "le sens général reste clair". Mais un étudiant universitaire qui va devenir un spécialiste de la langue et de la littérature française ne devrait en aucun cas, selon nous, obtenir une matière de langue française, étiquetée comme niveau A2, s'il commet systématiquement -ni même occasionnellement, dirions-nous- de

1 Dans le Volume complémentaire du CECR (2018: 182), les descripteurs de correction grammaticale à l'écrit sont reformulés mais le message reste le même: en ce qui concerne le niveau A2, l'apprenant "commet encore systématiquement des erreurs élémentaires" qui "peuvent parfois entraîner des malentendus"; au niveau B1, on "fait des erreurs occasionnelles que le lecteur peut généralement intterpréter grâce au contexte". 
simples erreurs d'accords en genre et en nombre, par exemple ${ }^{2}$. Pour ce type d'apprenants, la production écrite est fondamentale et ne devrait pas être reléguée à un plan simplement communicatif. L'étudiant universitaire doit donc savoir communiquer, certes, mais en démontrant un degré de maîtrise linguistique élevé ${ }^{3}$, suffisamment élevé pour ensuite transmettre une langue correcte à ses propres élèves.

En outre, nous sommes convaincu que ces étudiants, amenés à enseigner plus tard la langue, doivent acquérir des compétences particulières que d'autres apprenants, qui veulent simplement savoir communiquer en français, ne nécessitent aucunement. Ces compétences relèvent non seulement de la connaissance grammaticale de la langue étudiée, mais également de la capacité de comparaison avec leur propre système linguistique et d'explication des erreurs les plus fréquentes commises par les hispanophones. Une formation de ce type, comme le signale Doquin de Saint Preux, peut s'avérer être un véritable atout:

On ne peut s'empêcher de penser qu'il peut être utile à l'enseignant de langue française d'avoir conscience de ces phénomènes, de les connaître, de les comprendre, afin de devenir un meilleur "guide" dans le processus d'apprentissage de ses étudiants. Le comparatisme ne concerne donc pas directement les élèves, mais le professeur de français langue étrangère enseignant à un public hispanophone qui, par une réflexion sur les ressemblances et les différences de fonctionnement entre les deux langues saura tirer profit des effets de transfert et tentera de prévenir et de réduire les interférences. Cela implique, d'une part, une méthodologie et un apprentissage différents et adaptés à chaque communauté linguistique et, d'autre part, une analyse linguistique comparative à la base de la démarche pédagogique. (Doquin de Saint Preux, 2008: 15)

Par ailleurs, au-delà des raisons professionnelles, il ne faut pas oublier non plus que les élèves universitaires étudient une langue étrangère qu'ils sont constamment appelés à utiliser à l'écrit pour remettre travaux, examens et autres compositions propres du domaine académique et ce, dès les premiers niveaux. Ce ne sont donc pas des apprenants ordinaires et les lacunes linguistiques persistantes et récurrentes doivent faire l'objet d'un traitement particulier. Ce sont certaines de ces lacunes que nous voudrions modestement contribuer à éradiquer ${ }^{4}$, du moins en partie, grâce aux recherches que nous développons ici. Connaître précisément ces erreurs devrait permettre de prévenir leur enracinement, leur "fossilisation",

2 Certaines erreurs d'accords, comme celles du participe passé, appartiennent à un niveau plus avancé.

3 Le degré de maîtrise linguistique élévé auquel nous faisons référence se borne, bien entendu, aux contenus morphosyntaxique associés à chaque niveau. Nous n'avons nullement la prétention d'exiger, par exemple, à des apprenants faux débutants de maîtriser des structures complexes propres à des niveaux supérieurs.

4 Nous sommes pleinement conscient que la compétence parfaite n'existe pas et que les erreurs sont un atout pour l'apprenant dans son processus d'apprentissage de la langue étrangère, comme le rappellent Porquier et Frauenfelder (1980: 29). Cela n'empêche pas que l'apprenant et l'enseignant n'en tirent profit et ne tentent de les éliminer afin de parvenir, pour le premier, à s'exprimer de plus en plus correctement. 
pour utiliser le terme consacré, dans une interlangue, utile pour communiquer, certes, mais trop inachevée pour un certain public.

Nous nous concentrons dans cet article sur les fautes de langue les plus courantes commises par les apprenants universitaires hispanophones de Français Langue Étrangère (FLE) de niveau A2-B1 dans leurs productions écrites ${ }^{5}$. Or, la plupart des travaux du domaine de l'analyse des erreurs (Rodríguez Aguado, 2006: 179; Duchêne, 2013: 153; Doquin de Saint Preux et Sáez Garcerán, 2014; Flores Pérez, 2016: 82; Afifi, 2018: 286) révèlent que, quelles que soient la langue maternelle et la langue étrangère qui entrent en jeu, c'est le domaine de la morphosyntaxe qui est le plus touché. C'est la raison pour laquelle nous avons décidé d'y concentrer nos efforts, sans douter toutefois de l'importance des erreurs lexicales et orthographiques, que nous avons relevées, mais qui feront l'objet de travaux futurs. Nous présenterons le classement général des erreurs de morphosyntaxe les plus fréquentes et nous développerons ensuite un aspect particulier de ces erreurs, l'absence d'accord, qui présente la fréquence d'erreurs la plus élevée.

\section{L'analyse des erreurs et la didactique des langues}

Afin de réaliser notre étude, nous nous inscrivons dans la tradition de l'Analyse des Erreurs (AE) et partons des prémisses suivantes:

- les apprenants d'une langue étrangère sont influencés par leur langue maternelle (interférences externes) et ceux qui appartiennent à un même groupe linguistique, les hispanophones, par exemple, produisent inévitablement des erreurs caractéristiques et récurrentes dans leurs productions orales et écrites;

- les erreurs dues à des interférences internes, c'est à dire dues à une surgénéralisation ou une mauvaise interprétation des règles de la langue cible, sont moins prévisibles mais semblent se produire également dans des schémas récurrents;

- il est possible, grâce à une étude quantitative des productions écrites d'un groupe homogène d'apprenants, de dresser une liste des erreurs grammaticales les plus fréquentes commises par ces apprenants;

- grâce à une étude qualitative de ces erreurs les plus fréquentes, on pourra mener à bien un apprentissage ciblé de la langue étrangère, qui permettra aux apprenants, dans le meilleur des cas, de réduire à un pourcentage minimal

5 Les autres aspects de la compétence de production écrite, comme la cohérence et la cohésion, les erreurs pragmatiques ou sociolinguistiques, ne sont pas moins importants mais appartiennent à un domaine qui n'est pas exclusivement linguistique. À ce sujet, Rodríguez Aguado (2006: 178) nous rappelle que "si no se utilizan [los mecanismos cohesionadores] en las redacciones en lengua extranjera puede deberse a que se desconocen también en la materna. En este caso, las medidas pedagógicas para minimizar su importancia no afectan sólo a una asignatura". Dans tous les cas, ces différents aspects sont également intéressants et feront l'objet de futures recherches, tout comme la ponctuation, dont l'importance dans la communication écrite n'est plus à démontrer. 
leur production d'erreurs et d'acquérir une compétence grammaticale de base solide dans la langue cible.

Dans le domaine de la linguistique appliquée, la nécessité de prendre les erreurs des apprenants comme point de départ pour guider leur apprentissage de la langue étrangère remonte à la deuxième moitié du XX ${ }^{\mathrm{e}}$ siècle. Avant cela, Fries (1945: 9) et ensuite Lado (1957: 9) défendaient l'idée selon laquelle l'analyse contrastive de deux systèmes linguistiques permettrait de prédire avec exactitude les zones de difficultés et par là même les possibles erreurs des apprenants. Seulement, la pratique a démontré que nombre de celles-ci “n'étaient de toute façon pas prévues par les linguistes" (Corder, 1980: 9), certainement en partie parce qu'on n'avait pas pris en compte les interférences internes dues à l'influence du propre système de la langue cible, mais pas seulement. Ainsi, aux alentours des années 70, un certain nombre de linguistes ont démontré que différents facteurs sont responsables de l'apparition d'erreurs dans la production des apprenants de langue étrangère (Bhatia,1974: 339-341) et que la simple comparaison préalable avec la langue maternelle ne suffit pas, comme on le croyait auparavant, à construire un apprentissage ciblé sur les possibles zones de difficultés; seule une analyse en profondeur des erreurs effectivement commises par les apprenants permettrait de comprendre leurs causes, aussi diverses fussent-elles, et de développer des stratégies visant à les éviter.

Ainsi, l'AE comme technique pour améliorer l'enseignement/apprentissage des langues étrangères possède déjà une longue tradition mais n'a pas perdu de sa vigueur car, ces dernières années, les travaux -articles ou thèses de doctorat- publiés à ce sujet, qu'ils soient théoriques (Santos Gargallo, 1994; Alexopoulo, 2005; D’Aquino, 2016) ou pratiques, ont été nombreux, aussi bien que les publics et les langues étudiés: étudiants de FLE arabophones (Afifi, 2018), persanophones (Aghaeilindi, 2013), italophones (Jamet, 2013) ou turcophones (Delen Karaagaç, 2012); apprenants d'espagnol langue étrangère lusophones (Flores Pérez, 2016), francophones (Doquin de Saint Preux et Sáez Garcerán, 2014), croatophones (Kocman, 2011), grécophones (Palapanidi, 2011) ou anglophones (Madrid Fernández, 1999); apprenants hispanophones d'anglais langue étrangère (Rodríguez Aguado, 2006), etc.

Nous n'avons cependant trouvé qu'une seule référence à une publication sur les erreurs les plus fréquentes commises à l'écrit par les apprenants de FLE hispanophones ${ }^{6}$, celle menée à bien par Duchêne (2013) et qui porte sur les productions écrites d'étudiants d'une

6 Doquin de Saint Preux (2008) a bien réalisé une thèse doctorale dans laquelle elle présente, écrit et oral confondus, les erreurs caractéristiques des apprenants hispanophones de FLE mais ces erreurs ne sont pas présentées en termes de fréquence d'apparition. En outre, les erreurs d'accord n'y sont pas traitées.

Valverde Mateos (2012), pour sa part, s'est intéressée dans sa thèse aux erreurs commises à l'oral par les apprenants de FLE hispanophones. Nous comparons plus bas ses résultats avec les nôtres en ce qui concerne les erreurs d'accord. 
filière de Traduction et Interprétation ${ }^{7}$. Dans le classement proposé par l'auteure, les erreurs sont regroupées sur deux niveaux: par classe (syntaxe, lexique, orthographe grammaticale, orthographe lexicale, ponctuation et typographie) et par sous-classes (au sein de la syntaxe, par exemple: "choix fautif de la préposition", "choix du possessif", "choix du pronom relatif”, etc.) et les pourcentages sont effectués à partir de celles-ci. C'est un travail très intéressant qui nous fournit un panorama général des zones d'erreurs les plus fréquentes chez ce type d'étudiants.

En ce qui nous concerne, comme nous l'avons mentionné plus haut, nous concentrons nos efforts, en premier lieu, sur la morphologie et la syntaxe et nous nous attelons ensuite plus précisément au problème de l'accord puisqu'il regroupe le plus grand nombre d'erreurs dans notre classement. Nous ne pouvons, faute de place, analyser en profondeur les autres aspects problématiques les plus fréquents; ils feront l'objet de travaux futurs.

\section{Méthodologie suivie et corpus étudié}

Traditionnellement, on effectue une différence entre les erreurs de performance, simples fautes ponctuelles dues à diverses causes externes aux langues apprises, comme "des défaillances de mémoire, à des états physiologiques tels que la fatigue ou à des états psychologiques, comme par exemple une émotion forte" (Corder, 1980: 13), et les erreurs de compétence, ou erreurs proprement dites, dues à la compétence transitoire de l'apprenant. Il est, sans aucun doute, ardu -voire parfois impossible- de détecter à l'écrit, sans feedback de l'étudiant donc, si certaines erreurs apparaissent parce que l'élève ignore ou applique mal des règles de grammaire de la langue cible, ou simplement parce qu'il a rédigé trop vite, parce qu'il est stressé ou parce qu'il est influencé par n'importe quel autre facteur externe à sa connaissance effective de la langue.

En ce qui nous concerne, nous avons donc choisi de prendre comme point de référence la fréquence d'apparition de certaines erreurs pour les comptabiliser dans notre classement comme point de grammaire problématique à traiter afin d'améliorer les capacités en production écrite des apprenants concernés. C'est ce qui nous a amené à traiter le problème de l'absence d'accord, qui, très certainement, ne peut s'expliquer par une méconnaissance de quelques simples règles de grammaire en français.

Pour ce qui est des critères utilisés afin de classer les erreurs commises par les apprenants de langue étrangère, il en existe de nombreux, qui donnent parfois lieu à des classements extrêmement détaillés (Penadés Martínez, 2003-2004). Cependant notre but n'est pas de présenter une classification exhaustive en termes de descripteurs, mais plutôt une

7 D'autres études concernant la production écrite des étudiants de FLE hispanophones ont vu le jour mais elles ne s'occupent pas des erreurs spontanées puisqu'elles se basent sur un corpus de traduction de phrases qui comportent un certain nombre de difficultés prévoyant de possibles erreurs des sujets d'étude (Ramírez Gómez et Díaz González, 1995, 1996; Ramírez Gómez, 2000). 
étude claire des erreurs en elles-mêmes, pratique et qui puisse servir d'outil aussi bien aux enseignants qu'aux apprenants. Ainsi, partant du fait que nous nous intéressons uniquement aux erreurs de morphosyntaxe, les seuls critères que nous considérons utiles dans un processus d'identification/description, d'explication et de correction des erreurs sont les critères descriptif et étiologique: le critère descriptif nous permettra de distinguer les erreurs dues à l'omission, l'addition ou le mauvais choix d'un mot ou d'une forme, entre autres; le critère étiologique, auquel nous ne ferons appel que dans la partie plus détaillée dédiée au problème de l'accord, nous amènera à déceler les possibles sources des erreurs commises (interférence externe, interne ou autre).

Il est également bon de préciser que, au sein de notre étude, le domaine de la "morphosyntaxe" comprend aussi bien la morphologie que la syntaxe et le terrain d'intersection entre les deux, la morphosyntaxe proprement dite. Les erreurs que nous prendrons en considération seront donc les suivantes: les erreurs en rapport avec la construction de la phrase, comme le mauvais choix, l'omission ou addition d'un mot (préposition, déterminant, pronom, etc.), l'ordre des mots, un mauvais choix du temps ou du mode, les erreurs d'accord, les formes verbales et de déterminants erronées ou inexistantes, le phénomène de l'élision, etc.

Finalement, en ce qui concerne notre corpus, il est homogène dans la mesure où les 120 productions écrites que nous avons analysées sont le produit de 120 étudiant(e)s universitaires hispanophones de français langue étrangère appartenant à différentes promotions successives, de 18 à 20 ans dans leur majorité, et dont le niveau oscille entre A2 et B1, du fait de l'hétérogénéité de niveau existant au sein des classes de langue dans les premières années. Par ailleurs, ces productions écrites allant de 100 à 200 mots en moyenne ont été rédigées “à la maison". Enfin, dans le but d'analyser un échantillon représentatif de la compétence écrite du niveau signalé, nous ne nous sommes pas contenté d'un type d'écrit particulier et nous avons donc relevé les erreurs de textes variés, aussi bien en ce qui concerne le thème que la forme: textes descriptifs, plus majoritairement, mais aussi lettres de réclamation, courts récits au passé, faits divers, résumés de livres, textes d'opinion, etc.

\section{Résultats}

Parmi les 120 rédactions que nous avons examinées, nous avons relevé 902 erreurs de langue, que ce soit de morphologie ou de syntaxe. Nous présenterons maintenant les résultats de notre recherche de deux manières: nous allons premièrement exposer et commenter le classement général, dans lequel nous détaillons, en termes de quantité et de pourcentage, la répartition des erreurs au sein des grandes catégories, parties du discours ou règles grammaticales les plus touchées, et où nous fournissons, pour chaque item, un ou plusieurs exemples caractéristiques; ensuite nous analyserons de plus près le phénomène de l'absence d'accord 
et tenterons de fournir de possibles raisons à sa prépondérance au sein des productions écrites des étudiants de FLE hispanophones afin d'en réduire l'impact au moyen de traitements ciblées.

\subsection{Classement général}

Dans ce classement, nous avons délibérément effectué des choix de regroupement de certaines erreurs dans le but de présenter les résultats les plus cohérents possibles. Il reflète, selon nous, les aires d'erreurs les plus fréquentes commises par les hispanophones à l'écrit:

\begin{tabular}{lc}
\hline Quantité d'erreurs & Proportion \\
\hline Absence d'accord: 147 & \\
- Genre: 73 & \\
(5) *Une conversation très interesant & \\
- Nombre: 34 & $16,29 \%$ \\
(6) * Les changement & \\
- Personne verbale: 34 & \\
(7) *J'était très content & \\
- Accord du participe passé avec le COD placé avant le verbe: 6 & \\
(8) *Ils sont les meilleurs colocataires que j'ai eu &
\end{tabular}

Syntaxe des déterminants: 124

- Omission: 63

(9) *Le jeune couple avait problèmes

(10) *La famille le vit d'autre manière

(11) *Un désert de Marroque

(12) *Les jeunes écoutent musique

- Choix erroné: 37

(13) *Beaucoup des gens n'ont pas de travail

(14) *Il aime faire de sport

(15) *J'aimerais que tu viennes quelque jour

- Addition: 15

(16) *Il est professeur de la Biologie

— Déterminant incomplet: 9

(17) *Il y a beaucoup personnes 
Construction verbale et pronominalisation: 84

- Omission de préposition: 21

(18) *Il nous permet être informé

(19) *Ce n'est pas facile recommencer à étudier

- Choix erroné du pronom: 21

(20) *J'ai été ravi de lui voir

(21) *Je l'ai demandé une photo

— Addition de préposition: 18

(22) *Elle a proposé d'appeler à Lisa

— Choix erroné de préposition: 11

(23) *Si on s'approche à elle...

(24)*Elle s'intéresse par les langues étrangères

— Place du pronom complément: 8

(25) *Tu peux embrasser la

- Omission du pronom en: 3

(26) *Il y a quelques qui sont aussi célèbres

- Pronom complément double: 2

(27) * Carla lui a demandé au serveur...

Prépositions non régies par un verbe: 71

- Choix erroné: 64

(28) *Deux voitures circulaient sur une rue

(29) *J'ai vecu en une residence universitaire

(30) *Je suis allé à l'Inglaterre

Addition: 5

(31) *Je m'est allé à chez-moi

Omission: 2

(32) *Quelque chose original

Morphologie verbale: 68

(33) * La valise s'a ouvert

(34) *Ils pouvaint se permettre de vivre ensemble

(35) *J'ai conossai trop personne

Genre des substantifs: 56

(36) *Une jeune couple

(37) * La lit

$6,2 \%$

(38) *Un erreur

(39) * La seconde étage 


\begin{tabular}{lc}
\hline Quantité d'erreurs & Proportion \\
\hline Morphologie des déterminants: 48 & \\
$(40) *$ Les adolescents ne veulent pas se ressemblent à ses parents & \\
$(41) *$ Je peux dire que Lady Gaga est ma amie & $5,32 \%$ \\
$(42) * I l$ n'y a pas des concours récents & \\
$(43) *$ Annette s'intéresse à le monde de la musique & \\
\hline
\end{tabular}

C'est face à il est/elle est/etc.: 43

(44) ?Elle est la femme de ménage

(45) *...tu peux avoir [...] une femme avec autre ensemble comme 4,76\% couple et il est très fréquent

(46) *Mon père a 43 ans. C'est honnête et humble

Temps verbal (principalement, imparfait face à passé composé): 40

(47) ?*Un jour, Manuel obtenait un travail

$4,43 \%$

(48) ?*L'anglaise a eu des habitudes tres bizarres

Place des adverbes: 39

(49) *J'ai pensé qu'il vraiment t'aime

$4,32 \%$

(50) *Aussi, nous avons été en une fête

Omission du sujet pronominal: 26

(51) * Mais était une époque tres difficile

$2,88 \%$

(52) *Est étrange parler trois langues différents à la même fois

Élision: 26

(53) *La homosexualité

(54) *Je croyais que elle ne voulait pas

$2,88 \%$

(55) *Je etudie

Calques syntaxiques divers: 22

(56) *Elle est qui m'a reçu dans l'appartement

(57) *Chaque fois, les enfants commencent à voir avant la télé

Confusion qui/que: 18

(58) *C'est une chose que, pour nous, est normale

$1,99 \%$

(59) *Des couple avec travail ont un situation meilleur qui d'autres

Autres (21 items différents avec moins de 10 occurrences chacun): $90 \quad$ 9,97\%

Nous pouvons extraire plusieurs conclusions générales de ce classement. Tout d'abord, nous avons recensé 35 catégories différentes: les 14 premières, suffisamment représentées dans notre corpus pour prendre en compte leur pourcentage d'apparition, regroupent $90 \%$ des erreurs; les 21 restantes, rassemblées dans une section que nous avons nommée "Autres" du fait qu'elles sont peu représentatives, $10 \%$. Dans un premier temps, il nous semble donc nécessaire de considérer ces 14 premiers items comme prioritaires dans l'enseignement du 
français langue étrangère aux hispanophones, du moins pour ce qui est de la correction grammaticale:

1. Absence d'accord

2. Syntaxe des déterminants

3. Construction verbale et pronominalisation

4. Prépositions (non régies par un verbe)

5. Morphologie verbale

6. Genre des substantifs

7. Morphologie des déterminants

8. C'est face à il est/elle est/etc.

9. Temps verbal

10. Place des adverbes

11. Omission du sujet pronominal

12. Élision

13. Calques syntaxiques

14. Confusion qui/que

Par ailleurs, remarquons que le tiers de ces erreurs prioritaires $(30,03 \%)$ provient de deux sources principales: l'absence d'accord et la syntaxe des déterminants. Il ne fait aucun doute que ces deux zones grammaticales devront mériter une attention plus spéciale de la part des enseignants et des apprenants. Nous pouvons ensuite regrouper par importance deux autres groupes: un premier, dont chaque item représente entre 7 et $9 \%$ des erreurs totales, composé des problèmes ayant trait à la construction verbale, aux prépositions non régies par un verbe et à la morphologie verbale; un deuxième, dont les pourcentages oscillent entre 4 et $6 \%$, présente les erreurs de genre de substantif, de morphologie des déterminants, de confusion entre les formes c'est (ce sont) et il est (elle est, ils sont, elles sont), de temps verbal et de place des adverbes.

Finalement, moins représentées dans notre tableau mais d'une relative importance tout de même se trouvent les catégories suivantes: l'omission du sujet pronominal, l'élision non réalisée, des calques syntaxiques divers et la confusion entre les formes qui et que, pronoms relatifs mais aussi avec que conjonction de subordination. Les 21 items restants, parmi lesquels se trouvent, entre autres, les homophones grammaticaux, la syntaxe des adjectifs dans le syntagme nominal ou le mode verbal, ne présentent que peu d'occurrences et ne font donc pas partie, selon nous, des contenus prioritaires.

De nombreuses zones d'erreurs mériteraient un examen attentif afin d'en tirer les conclusions pertinentes et les applications didactiques correspondantes, cependant, faute de place, nous nous pencherons uniquement sur le phénomène de l'absence d'accord puisque c'est, de loin, l'erreur la plus répétée dans notre corpus. 


\subsection{L'absence d'accord}

Comme on peut le constater dans le tableau 1, l'absence d'accord est l'erreur principale que nous avons relevée dans les productions écrites des hispanophones étudiant le $\mathrm{FLE}^{8}$. Ces erreurs se répartissent en trois groupes principaux: accord de genre, accord de nombre et accord de personne. Les erreurs d'accord de genre représentent la moitié des erreurs d'accord dans notre corpus 9 : nous en avons relevé 73 , soit 49,65\% pour 147 erreurs au total. Les erreurs de nombre et de personne, quant à elles, sont moins nombreuses: nous en avons repéré 34 pour chaque catégorie, soit 23,12\%. Les six erreurs restantes, plus anecdotiques, concernent l'accord du participe passé avec le COD placé avant le verbe. Certainement ces dernières erreurs sont moins nombreuses tout simplement parce que cette construction syntaxique est rare dans notre corpus, d'autant plus que les apprenants concernés sont d'un niveau relativement bas et n'ont peut-être pas encore abordé ces constructions plus complexes.

La plupart des erreurs de genre et de nombre que nous avons relevées touchent des règles morphologiques simples: l'ajout, à la fin du mot concerné, d'un $e$ au féminin et d'un $s$ au pluriel; les erreurs d'accord de personne ne répondent pas à un schéma aussi simple. Par ailleurs, nous pouvons supposer que ces deux règles de base sont censées être connues des apprenants de niveau A2-B1. Pourquoi, dans ce cas-là, nous relevons autant d'erreurs d'accord au sein de notre corpus?

La première raison à laquelle nous pensons est qu'une proportion élevée d'étudiants ne réalise pas ou ne sait pas réaliser une relecture effective afin d'éliminer les erreurs d'accord évidentes. Cette compétence de relecture sera sans aucun doute un point à travailler avec les étudiants de FLE. Cependant, nous nous posons également les questions suivantes: pourquoi tant d'erreurs de ce type apparaissent en premier lieu et quelle peut être la possible raison de la prépondérance des erreurs d'accord touchant le genre?

Si l'on y regarde de plus près, on se rend compte que sur 73 erreurs d'accord de genre, 54 touchent des termes, déterminants ou adjectifs, qui auraient dû être accordés au féminin et, dans une majorité des cas, 49 sur 54, il s'agit de l'absence du e marquant le féminin; les six cas restants concernent des paires morphologiques de mots, pronoms ou déterminants. À cela, il faut ajouter que, sur les 49 occurrences mentionnées, nous recensons 44 exemples de non féminisation de mots terminés, au masculin, par consonne contre seulement cinq de mots terminés par voyelle. Or, l'ajout d'un $e$ à un mot terminé par consonne, on le sait, provoque

8 Chez Duchêne (2013: 153), en ce qui concerne son classement de 1'année 2010-2011, sur 431 erreurs de morphologie et de syntaxe, elle ne recense pas moins de 106 erreurs d'accord, soit $24,6 \%$ du total, ce qui prouve encore une fois l'importance de ce type d'erreur dans la production écrite des hispanophones.

9 Les erreurs que nous avons cataloguées comme erreurs d'accord de genre sont à différencier de celles touchant proprement la méconnaissance du genre lui-même des substantifs, comme dans les exemples (39) à (42). Certaines occurrences étaient ambiguës de ce point de vue-là mais nous avons tenté de prendre les décisions qui nous ont semblé les plus logiques. 
une modification au niveau de sa prononciation, ce qui n'est pas le cas des mots terminés par voyelle.

Valverde Mateos, au sein de sa thèse Analisis de errores de aprendientes de Francés Lengua Extranjera (FLE) basado en corpus orales (2012), remarque que l'une des erreurs les plus courantes chez les apprenants, tous niveaux confondus (de $\mathrm{A} 1$ à $\mathrm{C} 2$ ), est précisément celle de la concordance de genre: 9,1\% sur le total, sachant que cette auteure prend également en compte les erreurs de lexique; si elle ne considérait que les erreurs morphosyntaxiques, comme nous, ce pourcentage serait bien plus élevé. Son explication à ce phénomène est la suivante:

\begin{abstract}
El hecho de que otro de los errores más frecuentes se relacione con la concordancia del género, ha de observarse, sobre todo, siendo conscientes de que el género femenino en el francés requiere una pronunciación particular, que, en muchas ocasiones, por simplificación del discurso, tiende a omitirse. Aunque en muchos de los casos se trata de un error claro de competencia, como hemos señalado, encontramos otros donde el error es ambiguo y no nos permite dilucidar si el aprendiente es consciente de que está utilizando la forma femenina o no. Evidentemente, es posible que, en una producción escrita, el mismo aprendiente, por el tiempo de reflexión del que dispone y por la visualización de la palabra, no olvide la concordancia. (Valverde Mateos, 2012: 308)
\end{abstract}

Valverde Mateos considère que la prononciation "particulière" du féminin est à l'origine des erreurs de genres. La particularité de la prononciation des termes auxquels on ajoute un $e$ final au féminin vient certainement de ce que la consonne précédente, latente au masculin, doit alors s'articuler pleinement. Or, il n'est pas naturel, pour un hispanophone, de prononcer des consonnes occlusives - entre autres- finales, en position implosive donc, car ce n'est pas dans ses habitudes articulatoires. Cela ne veut pas dire pour autant que l'apprenant ne prononce pas la consonne finale "activée" par la féminisation, mais il est bien possible que, dans un certain nombre de cas, cette prononciation soit tellement peu appuyée qu'elle en devienne presque inaudible. Si on ajoute à cela le fait que, par l'influence de l'écrit et de mauvaises habitudes de lecture, les apprenants ont tendance à prononcer beaucoup de consonnes muettes du français, on confondra aisément, à l'écoute, les termes grand et grande prononcés indifféremment [grãd]. Et ce phénomène peut s'appliquer à un grand nombre de formes.

La présence d'une "forme phonétique unique" à l'oral amène alors l'auteure à se poser la question de savoir s'il s'agit parfois d'une erreur de compétence grammaticale ou bien de prononciation, en d'autres termes: est-ce que l'apprenant ne sait pas appliquer la règle de l'accord et/ou ne connaît pas la forme du féminin, ou bien est-ce qu'il ne prononce pas bien le terme qu'il aurait effectivement mis au féminin dans son discours?

Pour notre part, comme nous l'avons déjà signalé, nous ne croyons pas que l'apprenant de niveau A2, et encore moins B1, ignore la règle de l'accord ni soit incapable de former un féminin en ajoutant un simple $e$ final. Il faut donc probablement chercher la cause dans 
une prononciation défaillante. Cependant, les mêmes erreurs apparaissent à l'écrit. En effet, les exemples cités par Valverde Mateos comme étant caractéristiques du manque d'accord à l'oral coïncident avec un certain nombre des nôtres, appartenant cette fois à l'écrit. Voici les trois exemples de niveau B1 mis en avant et "transcrits" par Valverde Mateos (2012: 316317) pour illustrer las cas ambigus: "Dans un résidence", "Tout(s) les choses", "Je ne sais pas beaucoup de choses de la musique français".

En ce qui nous concerne, nous avons relevé, entre autres, 9 exemples de non féminisation de $u n$ et 6 exemples de tout, parmi les 44 mots terminés par consonne au masculin. Voici deux de ces exemples, dans lesquels l'étudiant connaît manifestement le genre de peau et de vie mais maintient un et tout au masculin:

\section{(60) * Sa peau est un peau bronzée \\ (61) * Tout la vie}

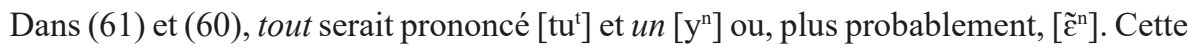
correspondance entre les erreurs relevées par Valverde Mateos et les nôtres nous invite donc à penser que la prononciation pratiquement indifférenciée du masculin et du féminin d'un certain nombre de mots pourrait être à l'origine d'un pourcentage non négligeable d'erreurs spontanées de "genre" à l'écrit. Il s'agirait donc d'erreurs essentiellement morphologiques, mais motivées par une prononciation/lecture déficiente. Paradoxalement, il se pourrait donc qu'un apprentissage plus ciblé et systématique de la différence de prononciation entre le masculin et le féminin favorise la diminution des erreurs d'accord de genre non seulement à l'oral, mais aussi à l'écrit.

Au sein des autres termes non féminisés terminés par consonne, nous n'avons recensé que 3 adjectifs qui prennent un $e$ au féminin en français mais dont le correspondant espagnol est épicène, ce qui pourrait expliquer l'absence d'accord en français. Il s'agit des exemples suivants:

(62) *Une bonne relation amical

(63) * La question social

(64) * La meilleur expérience

Pour ce qui est des erreurs d'accord de genre féminin touchant les paires morphologiques de mots, au nombre de 5, elles peuvent, cette fois, simplement répondre à des raisons de méconnaissance du paradigme des déterminants ou autres mots grammaticaux concernés, comme dans l'exemple suivant:

(65) *François a son jambe cassée 
En effet, il n'existe qu'une seule forme pour le masculin et le féminin singulier des adjectifs possessifs en espagnol alors qu'en français la forme varie selon le genre.

Finalement, les termes que l'on trouve au féminin alors qu'ils devraient être au masculin sont moins nombreux: nous n'en avons relevé que 19 cas sur 73. Certaines erreurs, comme la confusion entre les pronoms sujet $i l(s)$ et elle(s), par exemple, sont souvent le résultat de l'influence graphique et/ou phonique directe de formes espagnoles correspondantes:

(66) * Marie est mariée à Antoine, elles ont quattre enfants

(67) * Le seconde est le frère

(68) *Il est étudiante

(69) * Cela est très importante

(70) *Une homme

(71) *Dans un première lieu

En espagnol, les pronoms sujets de troisième personne, masculin ou féminin, singuliers ou pluriels, présentent tous la forme initiale el-, ce qui occasionne souvent une confusion chez les apprenants hispanophones, à l'oral également (Valverde Mateos, 2012: 305). Dans les exemples (67) à (71), il semblerait que les formes seconde, étudiante, importante, une et première puissent répondre d'une certaine manière à segundo, estudiante, importante, un, et primero.

Notre analyse de l'absence d'accord de genre nous a donc conduit à penser qu'une mauvaise prononciation et de mauvaises habitudes de lecture pourraient être à l'origine de bien des erreurs de ce type. Or, en ce qui concerne les erreurs d'accord de nombre et de personne, qui sont bien moins nombreuses que celles de genre, nous le savons, une influence phonétique pourrait également être à l'oeuvre. À ce sujet, Duchêne émet l'hypothèse suivante:

[...] les difficultés pour l'apprenant sont souvent dues au fait que les marques des catégories grammaticales et des fonctions, qui dans d'autres langues sont restées articulées, ne se prononcent pas en français; en espagnol hombres se distingue de hombre par l'ajout, en fin de mot, d'une consonne articulée au pluriel. En revanche, en français hommes est rigoureusement homonyme du mot homme. C'est l'article, qui dans de nombreux cas indiquera le nombre du mot: l'homme / les hommes. Ces observations sont également valables dans le cas des personnes du verbe. (Duchêne, 2013: 156)

C'est certainement le cas pour un grand nombre de nos exemples, comme les suivants: 
(72) *Ils étaient caste

(73) *Deux maison

(74) * Trois langue différents

(75) *Ils sont très amusant

En effet, la plupart des erreurs de nombre que nous avons recensées ont trait à l'absence du $s$ final: 26 sur 34. De nouveau, comme dans le cas des erreurs de genre, des erreurs de morphologie pourraient donc être dues en réalité à une influence phonétique.

Finalement, en ce qui concerne les erreurs d'accord de personne, elles se matérialisent surtout dans l'échange des désinences de la première et de la troisième personne du singulier, que ce soit au présent (*je t'écrit, *je peut) ou à l'imparfait (*j'achetait, *je me fâchait, *il étais, *quelqu'un me appelais), du fait peut-être de l'homophonie signalée par Duchêne et, dans le cas de l'imparfait, par l'influence de l'espagnol qui ne possède qu'une seule forme pour ces deux personnes, ou dans des accords sylleptiques abusifs comme *une jeune couple qui ont trois fils, *ma famille se composent de...

\section{Pratiques correctives}

Il y a 30 ans de cela, Santos Gargallo (1994: 172-173), dans un contexte d'enseignement différent, insistait déjà sur un point que nous avons signalé dans l'introduction de cet article: il existe des publics d'apprenants qui ont besoin d'atteindre un niveau de correction grammaticale élevé dans leurs productions écrites et pas seulement de savoir communiquer, comme c'est le cas des étudiants hispanophones d'études françaises, amenés à être des spécialistes de la langue.

Pour atteindre ce degré de correction, cette auteure propose deux techniques que nous pratiquons nous-même en classe et qui, pour notre part, fonctionnent. Grâce à leur mise en œuvre, l'amélioration des étudiants en terme de compétence grammaticale à l'écrit est patente, même au terme d'un semestre: d'une part parce que les étudiants connaissent mieux les zones principales de difficultés auxquelles ils sont confrontés et, d'autre part, parce qu'ils sont plus à même de repérer les erreurs dans leurs propres productions et de les corriger après relecture ${ }^{10}$. Ces techniques sont au nombre de deux: autocorrection, de la part des étudiants, de leurs propres productions écrites; sessions de correction d'erreurs réalisées à partir de phrases incorrectes des propres étudiants.

Pour améliorer l'autocorrection, Duchêne (2013: 158-159) pratique également une correction en plusieurs étapes et signale, à raison, que les étudiants doivent, en premier lieu, savoir comprendre et manier le métalangage grammatical employé pour décrire les erreurs.

10 Dans le contexte des erreurs caractéristiques des apprenants de FLE hispanophones à l'oral, Tomé Díaz (2018: 151) considère également que "el descubrimiento de los errores por el alumno es esencial para desarrollar la autoconsciencia, la reflexión y la corrección". 
Ceci est fondamental, non seulement pour comprendre les symboles de l'enseignant, mais également parce que le savoir grammatical et linguistique sur la langue permet, comme nous le signalions au début de l'article, de fournir des explications plus satisfaisantes à de futurs apprenants.

Par ailleurs, les symboles utilisés pour signaler les erreurs doivent être simples et fonctionnels. La démarche d'autocorrection que nous pratiquons nous-même est la suivante: tout d'abord, nous signalisons, grâce à un système de notation simple, les erreurs sans les corriger et rendons ensuite la copie à l'étudiant pour qu'il tente de les corriger lui-même; l'étudiant remet alors une deuxième version de sa rédaction que nous corrigeons finalement de nouveau et rendons à l'étudiant afin qu'il puisse évaluer son degré de maîtrise en matière de connaissance, identification et correction de ses propres erreurs. Cette pratique demande un travail double à l'enseignant mais se révèle être très formatrice pour l'étudiant.

Les sessions de correction collective, quant à elles, ont ceci de bénéfique qu'elles permettent de sélectionner les erreurs les plus courantes et de faire réfléchir toute la classe sur leurs possibles sources et corrections. Dans notre cas, dans les premières sessions de correction, nous signalons les erreurs et les étudiants n'ont qu'à réfléchir pour savoir en quoi elles consistent et comment les corriger; puis, dans les sessions suivantes, lorsque les étudiants ont progressé car ils ont rencontré de nombreuses fois des erreurs caractéristiques, nous les laissons déceler les erreurs avant de procéder à leur description et possible correction. Dans l'idéal, nous essayons de sélectionner des phrases dont les erreurs sont fréquentes et sont en rapport avec les contenus grammaticaux étudiés à l'époque donnée.

Ce système progressif vise à amener l'étudiant à être capable de repérer par lui-même, en fin de parcours, les erreurs qu'il aurait pu commettre dans ses productions écrites. Cela nous conduit tout naturellement à la technique complémentaire de l'autocorrection: la relecture.

Tout au long de notre article, nous avons vu qu'il existe un certain nombre de catégories grammaticales plus touchées que d'autres à l'écrit, avec une prépondérance des erreurs d'accord. Pour ces dernières, nous avons émis l'hypothèse selon laquelle un travail spécifique sur la prononciation des apprenants pourrait en réduire fortement l'apparition spontanée. Cependant, en ce qui concerne les erreurs que l'apprenant continuerait de commettre sans réfléchir, il est possible de travailler également la technique de la relecture, peu ou pas du tout pratiquée par les étudiants et souvent non maîtrisée.

La relecture est un exercice difficile car il demande, entre autres, une capacité d'analyse syntaxique qu'un certain nombre d'étudiants universitaires de FLE ne domine pas, ou tout au moins, ne sait pas appliquer à l'apprentissage d'une langue étrangère. Grâce à cette technique et avec une connaissance de certaines règles de base, un grand nombre d'erreurs 
spontanées pourraient être corrigées. C'est le cas pour les erreurs d'accord, mais également pour des construction diverses: omission du sujet pronominal, omission d'un déterminant, mauvais choix du pronom complément selon la construction verbale, etc.

\section{Conclusions}

S'il est vrai que les années d'expérience fournissent souvent aux enseignants une connaissance des erreurs typiques que commettent à l'écrit -et à l'oral- leurs étudiants de langue française hispanophones, cette connaissance n'est pas forcément systématisée et nous espérons que notre classement, rigoureux et plus précis du fait qu'il se base sur des calculs de fréquence et porte uniquement sur la morphosyntaxe, pourra servir d'outil de consultation dans le but d'aborder l'enseignement/apprentissage du français de manière plus ciblée, du moins en ce qui concerne la production écrite dans les premiers niveaux.

En effet, comme le défend Doquin de Saint Preux (2008: 15), un apprentissage ciblé du français pourrait contribuer à réduire en grande partie ces erreurs avant qu'elles ne se fossilisent dans l'interlangue de l'apprenant. Or, la plupart des manuels de FLE ne prennent pas en compte les particularités langagières des étudiants auxquels ils s'adressent. Il revient donc à l'enseignant d'adapter sa méthode et ses contenus à son public afin d'engendrer chez lui un haut niveau de correction grammaticale. N'oublions pas que nous faisons référence ici à un public de spécialistes de la langue étrangère, qui ne peuvent se permettre de simplement "se faire comprendre" dans la langue en question.

Pour ce qui est des erreurs les plus fréquentes, on devra particulièrement faire attention à celle de l'accord, car c'est la plus répandue dans notre corpus. À ce sujet, nous avons comparé nos résultats avec ceux de Valverde Mateos (2012), qui s'est occupé pour sa part des erreurs commises à l'oral, et nous avons émis l'hypothèse selon laquelle une meilleure maîtrise de la langue orale et une connaissance plus approfondie de la (non)correspondance entre code écrit et code oral, complétées ensuite par l'acquisition des techniques de relecture et d'autocorrection, permettrait aux apprenants de commettre moins d'erreurs d'accord. Cette hypothèse est encore à confirmer dans un futur travail.

\section{Références bibliographiques}

AfIFI, Dalia. 2018. "L'analyse des erreurs en production écrite chez les apprenants arabophones en classe de FLE" in Anales de Filología Francesa, no 26, 279-293: < https://revistas. um.es/analesff/article/view/analesff.26.1.352441/253861> [12/04/2021].

AghaeILINDI, Somayé. 2013. La pédagogie de l'erreur en production écrite dans l'apprentissage du française langue étrangère, chez les étudiants persanophones Thèse doctorale, Université Toulouse 2-Le Mirail: <https://tel.archives-ouvertes.fr/file/index/docid/973497/ filename/ Aghaeilindi_Somaye.pdf $>$ [12/04/2021]. 
Alexopoulo, Angélica. 2005. “Aproximación al tratamiento del error en la clase de E/LE desde la perspectiva del análisis de errores" in Estudios de Lingüística Aplicada, no 41, 102 125: <https://ela.enallt.unam.mx/index.php/ela/article/view/ 640/719> [10/04/2021].

BÉRARD, Evelyne. 1991. L'approche communicative. Théorie et pratiques. Paris, Clé International.

Bhatia, Aben T. 1974. "An Error Analysis of Students' Compositions” in IRAL: International Review of Applied Linguistics in Language Teaching, $\mathrm{n}^{\circ}$ 12/4, 337-350.

Cadre européen commun de référence pour les langues: apprendre, enseigner, évaluer. 2001. Strasbourg, Unité des politiques linguistiques: $<$ https://rm.coe.int/16802fc3a8 $>$ [12/04/2021].

Cadre européen commun de référence pour les langues: apprendre, enseigner, évaluer. Volume complémentaire avec de nouveaux descripteurs. 2018. Strasbourg, Division des politiques éducatives. Service de l'Éducation: $<$ https://rm.coe.int/cecr-volume-complementaire-avec-de-nouveaux-descripteurs/16807875d5> [12/04/2021].

Corder, S. Pit. 1980. “Que signifient les erreurs des apprenants?” in Langages, n 57, 9-15.

D’Aquino, Alessandra. 2016. “¡De los errores se aprende! La corrección como instrumento didáctico" in Textos Didáctica de la Lengua y de la Literatura, n 71, 7-13.

Delen CaragaÇ, Nurcan. 2012. "Des erreurs linguistiques et des effets de contexte: analyse de differents types d'erreurs commises par les etudiants turcs" in Uluslararasi Sosyal Arastirmalar Dergisi. The Journal of International Social Research, $\mathrm{n}^{\circ}$ 5/23, 175-184: <https://www.sosyalarastirmalar.com/cilt5/cilt5sayi23_pdf/delen_nurcan.pdf> $[12 / 03 / 2021]$.

Doquin de Saint Preux, Anne. 2008. L'enseignement du français aux hispanophones. Problèmes repérés, études linguistiques, propositions didactiques. Thèse doctorale, Université Paris IV.

Doquin de Saint Preux, Anne; Sáez Garcerán, Patricia. 2014. “Análisis de errores en dos tipos de producciones escritas de alumnos franceses de ELE de nivel avanzado" in Revista Nebrija de Lingüística Aplicada a la Enseñanza de las Lengua, $\mathrm{n}^{\circ} 17$. <https://revistas. nebrija.com/revista-linguistica/article/view/247/215> [24/03/2021].

DuchêNe, Nadia. 2013. "La compétence rédactionnalle en langue française du futur traducteur hispanophone" in Çédille, revista de estudios franceses, $\mathrm{n}^{\circ} 9,145-160$. $<$ https://cedille. webs.ull.es/9/09duchene.pdf $>$ [25/04/2021].

Flores PÉREZ, Tamara. 2016. "El progreso en el error: análisis de la producción escrita de alumnos lusófonos" in SAInz GARCía, Ángel M. (dir.). El español como lengua extranjera en Portugal II: retos de la enseñanza de lenguas cercanas. Ministerio de Educación, Cultura y Deporte, 80-89.

FRIES, Charles C. 1945. Teaching and Learning of English as a Forein Language. Michigan, University of Michigan Press.

JAMET, Marie-Christine. 2013. "La complexité linguistique est-elle complexe pour l'apprentissage? Nature et interprétation des erreurs, et en particulier des erreurs de syntaxe dans des productions écrites d'apprenants italophones du français" in PAPROCKA-PIOTROWSKA, Urszula et al. (éds). La complexité en langue et son acquisition, LUBLIN, Towarzystwo 
Naukowe KUL \& KUL (Società delle scienze dell'università cattolica di Lublin). Lublin, Wydawnictwo Werset, 99-104. <https://www.kul.pl/files/60/la_complexite_programme. pdf $>[11 / 04 / 2021]$.

Kocman, Ana. 2011. "Errores gramaticales comunes en alumnos croatas de español como lengua extranjera en la producción escrita" in MarcoELE: Revista de Didáctica Español Lengua Extranjera, $\mathrm{n}^{\circ}$ 13: < https://marcoele.com/descargas/13/kocman-errores_croatas.pdf $>$ [12/04/2021].

LAdo, Robert. 1957. Linguistics Across Cultures. Michigan, University of Michigan Press.

MAdrid Fernández, Daniel. 1999. “Errores gramaticales en la producción escrita de los angloamericanos" in Romero, Antonio et al. (éds). Educación lingüística y literaria en el ámbito escolar: actas del I Congreso Internacional sobre Educación Lingüística y Literaria en el Contexto del Sistema Educativo. Celebrado en Granada, del 21 al 23 de mayo de 1998. Grenade, Grupo Editorial Universitario, 609-626: <http://www.ugr.es/ dmadrid/Publicaciones/ Errores \%20Americanos.pdf> [12/04/2021].

PALAPANidi, Kiriakí. 2011. "Los errores léxicos intralinguales semánticos en la producción escrita de los aprendientes griegos de español" in Revista Nebrija de Lingüística aplicada a la enseñanza de Lenguas, $\mathrm{n}^{\circ}$ 9: <https://www.nebrija.com/revista-linguistica/los-errores-lexicos-intralinguales-semanticos-en-la-produccion-escrita-de-los-aprendientes-griegos-de-espa\%C3\% B1ol.html $>[12 / 04 / 2021]$.

Penadés Martínez, Inmaculada. 2003-2004. "Las clasificaciones de errores lingüísticos en el marco del análisis de errores" in Linred: Lingüística en la Red, $\mathrm{n}^{\circ}$ 1: <http://www.linred. es/informacion_pdf/informacion3_250204.pdf > [22/05/2021].

Porquier, Rémy; Frauenfelder, Ulrich. H. 1980. “Enseignants et apprenants face a l'erreur, ou de l'autre côté du miroir" in Le français dans le monde, n 154, 29-36.

Puren, Christian. 2006. "De l'approche communicative à la perspective actionnelle" in $\mathrm{Le}$ français dans le monde, $\mathrm{n}^{\circ} 347,37-40$.

Ramírez Gómez, Carmen. 2000. "Elaboración de un catálogo de dificultades gramaticales francés-español, español-francés" in ORTEGA RomerA, Andrés et al. (coords). II Jornadas Andaluzas de Calidad en la Enseñanza Universitaria. Desarrollo de Planes de Calidad para la Universidad. Materiales para la Calidad. Universidad de Sevilla, 65-87.

Ramírez Gómez, Carmen; Díaz González, Pedro. 1995. “Gramática contrastiva: Una experiencia de innovación en el aula" in Revista de Enseñanza Universitaria, $\mathrm{n}^{\circ}$ 10, 47 66.

Ramírez Gómez, Carmen; Díaz González, Pedro. 1996. “Análisis contrastivo y análisis de errores" in Alonso, Emilia et al. (éds). La lingüística francesa: gramática, historia, epistemología. Séville, Universidad de Sevilla, Tome II, 329-337.

Rodríguez Aguado, José I. 2006. “Análisis de errores en la cohesión textual. La referencia” in ES: Revista de filología inglesa, n 27, 175-188.

SANTos Gargallo, Isabel. 1994. "Análisis de errores: valoración gramatical y comunicativa en la expresión escrita de estudiantes de ele. Una propuesta didáctica" in MonTEysA PEDRÓ, Salvador \& Antonio Garrido Moraga (éds). Actas del Segundo Congreso Nacional de 
Anales de Filología Francesa, n. ${ }^{\circ}$ 29, 2021

MARC VIÉMON

ASELE español para extranjeros: didáctica e investigación. Madrid, del 3 al 5 de Diciembre de 1990. Málaga: ASELE, 169-174.

Tomé DíAz, Mario. 2018. "Sistema de faltas de pronunciación y corrección fonética en un corpus oral FLE” in Revista de Lingüística y Lenguas Aplicadas, n 13, 145-155.

Valverde Mateos, Ana. 2012. Análisis de errores de aprendientes de francés lengua extranjera (FLE) basado en corpus orales. Thèse doctorale, Universidad Autónoma de Madrid: $<$ https://repositorio.uam.es/ handle/10486/10147?show=full $>[21 / 05 / 2021]$. 
